\title{
La dimensión social de la Infraestructura Verde. Una revisión sobre el bienestar socioambiental en el espacio metropolitano
}

\section{The social dimension of Green Infrastructure. A review of socio-environmental well-being in the metropolitan space}

\author{
Ricardo Iglesias Pascual' [1] y Francisco José Gómez García² (1)
}

\begin{abstract}
RESUMEN
Los estudios que abordan la dimensión social de los espacios que albergan la función ecológica en el ámbito urbano se han centrado fundamentalmente en el análisis local de los espacios verdes. Sin embargo, la dimensión social de las Infraestructuras Verdes (IV) como conjunto de espacios libres a escala metropolitana ha sido un aspecto poco analizado. Este artículo a partir de una metodología de búsqueda inmersiva de 123 documentos y 539 casos de estudios desarrolla una revisión crítica de los principales aspectos analizados sobre el impacto social de la IV. Nuestros resultados muestran desde el punto de vista metodológico, como el predominante carácter cualitativo y local de estos estudios, dificulta generalizar sus resultados a escala metropolitana. A su vez, la creciente complejidad del espacio urbano hace necesario focalizar el análisis de la dimensión social de la IV desde la escala metropolitana, prestando especial atención a las percepciones ciudadanas y una gestión de los espacios verdes más descentralizada de cara a asegurar el bienestar socioambiental ciudadano.
\end{abstract}

Palabras clave: Infraestructura verde; bienestar socioambiental; espacio metropolitano

\section{ABSTRACT}

The studies that analyse the social dimension of the spaces that support the ecological function in the urban environment have focused fundamentally on the local analysis of green spaces. However, the social dimension of Green Infrastructures (IV) as a set of free spaces on a metropolitan scale has been little analysed. This article, based on an immersive search methodology of 123 documents and 539 case studies, develops a critical review of the main aspects analysed on the social impact of the spaces that make up the IV. Our results show from a methodological point of view how the predominant qualitative and local character of these studies makes it difficult to generalise their results to a metropolitan scale. Given the growing complexity of urban space, it is necessary to focus on analysing the social dimension of the IV from the metropolitan scale, paying special attention to citizens' perceptions and a more decentralised management of green spaces in order to ensure the socio-environmental wellbeing of citizens.

Keywords: Green infrastructure; socio-environmental well-being; metropolitan space 
El concepto de Infraestructura Verde (IV a partir de ahora) es una perspectiva de ordenación del espacio libre que asume como principios, por un lado, la integración en red de aquellos elementos de especial valor ambiental dentro de un territorio, y por otro, la potenciación de su multifuncionalidad a través del impulso de los servicios ecosistémicos (Baxendale \& Buzai, 2019, Lovell \& Taylor, 2013). Frente a un enfoque más tradicional de ciudad y naturaleza como elementos opuestos y excluyentes, la IV supone la búsqueda del equilibrio entre los componentes artificiales y naturales del territorio (Newell et al., 2013), especialmente en sistemas metropolitanos, con el objetivo principal de aporta servicios ecológicos, económicos y sociales mediante soluciones naturales (Cantó, 2014). A su vez, este carácter multifuncional y holístico, al igual que convierte la IV en un instrumento clave para un planeamiento más sostenible con el que orientar el crecimiento urbanístico dentro los planes de ordenación territoriales (Aguilera et al., 2018), dificulta su definición y su implementación en la planificación territorial (Feria Toribio \& Santiago Ramos, 2017).

A su vez este papel clave de la IV en la planificación territorial adquiere una mayor trascendencia si se tiene en cuenta que la red de espacios que conforman la IV de cualquier área urbana o metropolitana, además de su evidente dimensión ambiental y ecológica, tienen una gran importancia de cara a proporcionar experiencias de bienestar social a los residentes (Richards \& Thompson, 2019) que permite minimizar los efectos negativos de vivir en el entorno construido.

De este modo se puede considerar que existe una relación holística entre los espacios abiertos que forman las IV (Kim \& Song, 2019) y una gama de actividades sociales que facilita la capacidad de los habitantes metropolitanos para recrear, socializar y realizar otras actividades individuales y grupales regulares fuera de sus hogares (Selman, 2009). Es en estos espacios donde en ocasiones la población local desarrolla importantes relaciones sociales y familiares, actividades fundamentales en la vida cotidiana, en lugar de los parques distantes y los paisajes excepcionales lejos de casa (Sullivan, Kuo \& De Pooter, 2004). En este sentido existe un amplio consenso respecto a la importancia de la IV a la hora de tener contacto con la naturaleza, disfrutar de la belleza paisajística, la realización de actividades de ocio y juego, o el desarrollo de interacciones sociales entre otros ejemplos (Brown et al., 2014).

Sin embargo, frente al amplio estudio desarrollado en la literatura académica sobre su dimensión ecológica, urbanística y ambiental, la dimensión social ha sido un aspecto mucho menos abordado en las investigaciones e informes internacionales disponibles (Figura 1).

Figura 1.

Distribución de temáticas analizadas sobre la IV en la bibliografía académica

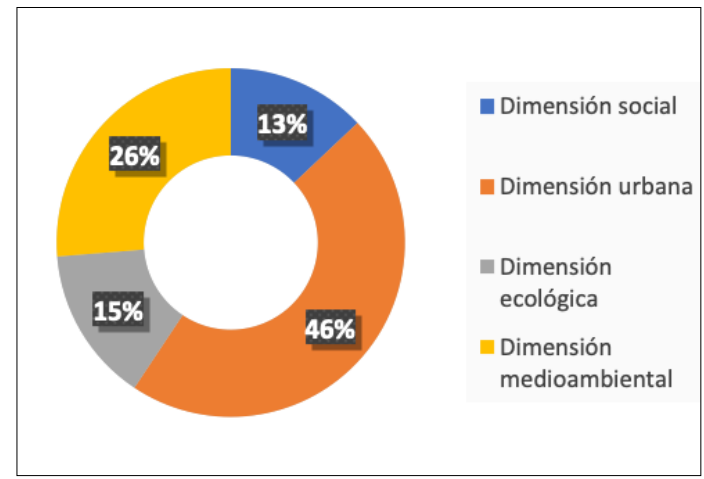

Fuente: Elaboración propia 
Este vacío en el estado de la cuestión se acrecienta aún más si se tiene en cuenta que la gran mayoría de las investigaciones se centran en analizar la dimensión social de la IV focalizan su enfoque especialmente las relaciones entre el uso de parques o zonas verdes urbanas en sus diferentes escalas pero especialmente en los parques de barrio siempre dentro de una escala local o infralocal (i.e. Pirnat \& Hladnik, 2019; Zwierzchowska et al., 2019) Resulta evidente que no todos los componentes que se agrupan dentro del concepto de la IV tienen una dimensión o impacto social, en el sentido de ejercer un efecto positivo sobre el bienestar socioambiental y el incremento de la cohesión social en la ciudad (Martí et al., 2020; van Vliet, K. \& Hammond, 2020). Sin embargo para abordar la diversa naturaleza de sus componentes que si tienen un uso potencialmente social hay que trabajar una escala territorial superior a la local para conseguir abordar la distribución espacial de estos componentes en el espacio metropolitano (Dipeolu, Ibem \& Fadamiro, 2020; Zwierzchowska et al., 2019).

Por ello se hace necesario revisar las temáticas y las características de la metodología desarrollada a la hora de analizar la relación entre los elementos espaciales que componen las IV y la satisfacción social. Un aspecto cómo la valoración y satisfacción social sobre el uso de un espacio, aspecto dónde la subjetividad social juega un papel crucial, puede verse determinado en su estudio por los instrumentos metodológicos puestos en juego y los temas que se planteen en la investigación. En gran medida estos instrumentos determinan que se desarrolle un análisis de carácter interpretativo y descriptivo o se desarrollen interpretaciones que planteen la causalidad entre las variables analizadas

Así en el presente estudio, en primer lugar, se ha desarrollado una revisión, a partir de una búsqueda inmersiva sobre la literatura académica que analiza la dimensión e impacto social de los elementos que componen las IV. A partir de esta revisión se han analizado tres aspectos fundamentales de estas investigaciones: i) los principales focos temáticos que abordan la relación entre el factor ecológico y social; ii) la escala territorial aplicada y por último, iii) los instrumentos y estrategias metodológicas puestas en marcha. Posteriormente, a partir de los resultados se ha planteado una discusión sobre las características generalizables de estos estudios para finalizar reflexionando sobre los aspectos a tratar a la hora de conseguir análisis socioterritoriales que permitan construir propuestas de planificación y diseño de las IV que atiendan al desarrollo del bienestar social a escala metropolitana, superando así el ya comentado tradicional enfoque local e infralocal que caracteriza mayoritariamente estos estudios.

\section{Metodología}

En primer lugar, siguiendo las fases de Kitchenham y Charters (2007), se ha desarrollado una revisión sistemática de la literatura. La búsqueda de información general se realizó entre enero y febrero de 2019, empleando fundamentalmente los términos de "Infraestructura verde" y "dimensión social", con el objetivo inicial de estimar el volumen de la producción disponible. Para ello se ha realizado un sondeo en varios idiomas en los principales navegadores web: Google, Mozilla Firefox y Microsoft Edge. A razón de seleccionar la literatura que sirve de base a los objetivos planteados se ha realizado una búsqueda inmersiva o a la inversa dentro de los principales buscadores y recursos disponibles on-line para la investigación científica. Para ello se parte de una búsqueda de información general y superficial de carácter generalista, para focalizar posteriormente en una búsqueda de publicaciones específica con un perfil concreto y alojadas en determinados repositorios de corte académico y científico. 
La búsqueda de información general se realizó entre enero y febrero de 2019, a partir de la búsqueda de la producción del término "Infraestructura verde" y en qué lengua materna se encontraba el grueso productivo. Tras esta fórmula de búsqueda, cómo en otras estudios (Salvador-Oliván, Marco-Cuenca \& Arquero-Avilés, 2018), se constató que la mayoría de ellos eran publicaciones indexadas en determinados motores de búsqueda, repositorios y redes especializadas en contenido científico y en bibliografía académica como es el caso de Google Scholar, ResesearchGate, Science Direct, Scientific Research, Dialnet, MDPI y Publons. Tras esta operación, resultó evidente que la mayoría de resultados están desarrollados en inglés, ocupando el español un segundo lugar destacado respecto a otros idiomas como el francés. Tras esta primera consulta, se indagó sobre la producción científica inglesa e hispana de forma más concreta. A tal fin realizamos varias búsquedas duales empleando palabras claves, en inglés y en español, relacionadas con el campo semántico del término "infraestructura verde".

Al seguir siendo los resultados devueltos muy numerosos se concretó el algoritmo de búsqueda con operadores booleanos y comandos para acotar la respuesta recibida por los navegadores (una tipología documental determinada, un formato de archivo específico, un periodo temporal de producción bien definido), descartando de este modo el máximo de ruido posible (publicaciones de blogs, foros, redes sociales no académicas, prensa...).

Una vez localizados los principales repositorios, a partir de la técnica de bola de nieve (Greenhalgh \& Peacock, 2005) se ampliaron el número de investigaciones abordadas. Todo este proceso nos permitió seleccionar 110 investigaciones, basadas en 523 casos de estudios que suponen la base de la presente revisión. Cabría destacar, que estas investigaciones han mostrado un crecimiento constante y generalizado desde 2008 a nivel internacional. De hecho el $32 \%$ están centradas en casos europeos, el $35 \%$ asiáticos, el $18 \%$ norteamericanos y el $6 \%$ sudamericanos (Figuras 2 y 3). A partir de estos casos se ha hecho una compilación de las características de los tres principales aspectos que se indicaron al inicio de la presente investigación: la metodología de análisis, la escala geográfica de los estudios y las principales temáticas abordadas en la relación IV y satisfacción social.

Figura 2.

Evolución de la producción científica sobre la IV (2008-2020)

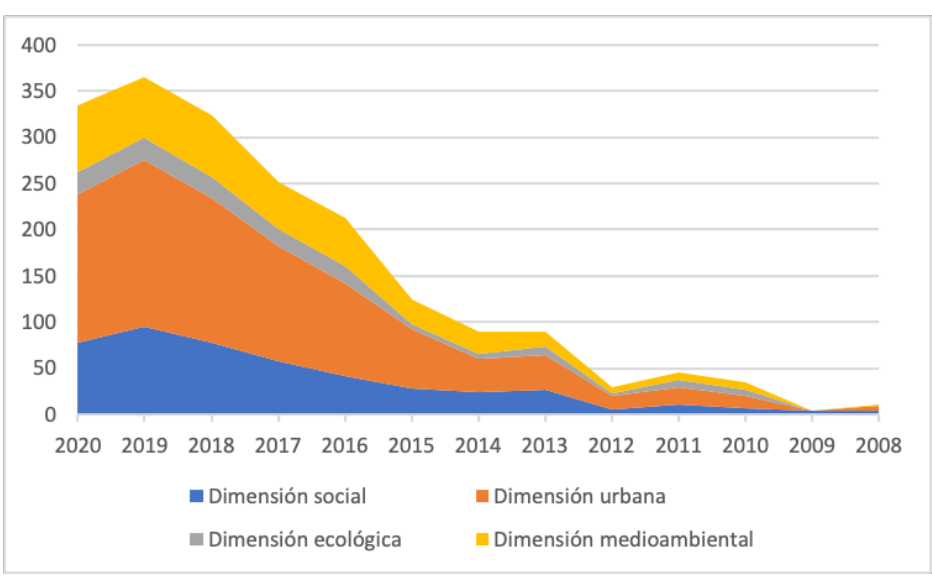

Fuente: Elaboración propia 
Figura 3.

Distribución territorial de los casos de estudio en IV

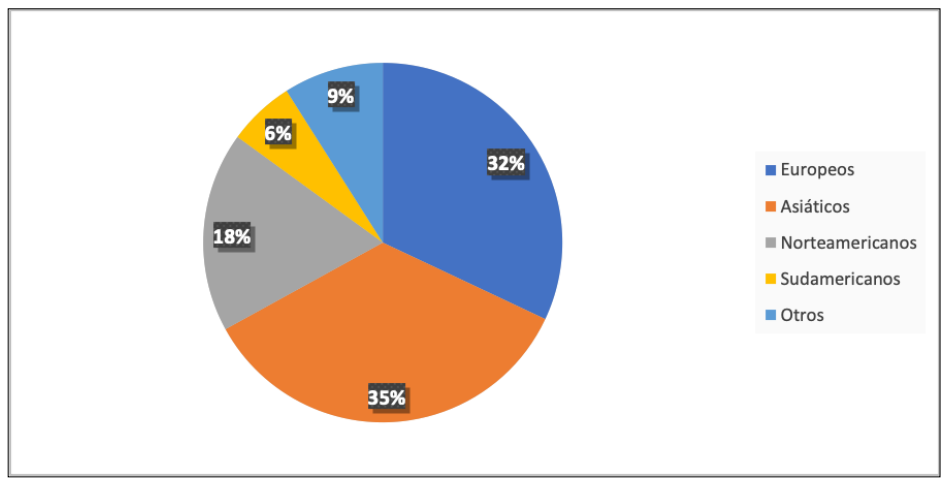

Fuente: Elaboración propia

\section{Metodologías y escalas de análisis}

\section{Antecedentes metodológicos}

A la hora de sintetizar la tipología de análisis puestos en marcha por las investigaciones que han sido utilizadas como base del estudio, se puede considerar que son esencialmente tres las metodologías desarrolladas: (i) estudios de carácter exclusivamente cuantitativos (16\%), (ii) estudios cualitativos (57\%) e (iii) investigaciones de carácter mixto que alternan ambas estrategias (27\%). Evidentemente, en numerosos casos las diferentes estrategias desarrolladas no resultan contrapuestas sino complementarias, según las características del estudio, los objetivos propuestos y los diferentes aspectos de las percepciones y actitudes sociales que sobre la IV se pretende analizar.

Cabe destacar como en las investigaciones desarrolladas sobre la relación entre las zonas verdes, el desarrollo de la actividad física y el bienestar social, la puesta en práctica de metodologías cuantitativas tienen un importante papel (i.e. Kaczynski \& Henderson, 2007; Lamond, J \& Everett, 2019). Si bien estos estudios han ofrecido una visión global sobre este tema, gran parte de los resultados más descriptivos y esclarecedores sobre la influencia de las zonas verdes es fruto de investigaciones de origen mixto (i.e. Librett et al., 2007). De hecho diversas revisiones bibliográficas (i.e. Rakhshandehroo et al., 2015; Venkataramanan et al., 2019), consideran que es esta metodología la más adecuada a la hora de desarrollar un análisis más profundo de las implicaciones sociales de las infraestructuras y los espacios verdes. Estos estudios de carácter mixto o mixed-methods, utilizan dinámicas de carácter cuantitativo y cualitativo conjuntamente, alternando la realización de grupos de discusión, entrevistas en profundidad, junto a cuestionarios en una búsqueda de una mayor representatividad muestral para dar un aval de representatividad estadística a los resultados obtenidos (i.e. Kazmierczak, 2013).

Los estudios basados en métodos estrictamente cualitativos, ya sea a través de entrevistas individuales en profundidad, en grupos focales, observación directa u observación participante, ofrecen unos resultados que complementan los hallazgos cuantitativos y aportan importantes 
contribuciones al análisis sobre la influencia que tienen los espacios verdes en las percepciones sociales colectivas e individuales (i.e Anguelovski, Irazábal-Zurita \&. Connolly, 2019). En particular los instrumentos cualitativos permiten aclarar o matizar en algunos casos los resultados en ocasiones contradictorios que ofrecen las investigaciones cuantitativas.

Por otro lado, dentro de los estudios que utilizan métodos exclusivamente cualitativos destacan aquellos que se basan en el uso de las entrevistas, ya sean en profundidad o mediante cuestionarios estándar (Kaczynski \& Henderson, 2007). En esta misma línea se encuentran investigaciones que alternan las entrevistas con la observación directa (Peters, Elands \& Buijs, 2010). Estas estrategias metodológicas son especialmente adecuadas para analizar de una marea global la subjetividad social que genera cada individuo sobre el uso del espacio verdes y muy especialmente analizar los tipos de interacciones sociales que se desarrollan, aspecto esencial para dar orientaciones a los planificadores y gestores sobre el papel y características de estos ámbitos territoriales deben tener para aumentar el grado de cohesión social de una comunidad (Swapan, Iftekhar \& Li, 2017). Dentro de esta misma línea metodológica se pueden encontrar estudios cualitativos los que priorizan la realización de grupos de discusión sobre la entrevista a la hora de recoger la información (Barnhill \& Smardon, 2012).

De este modo se puede considerar que si bien la investigación cuantitativa ha contribuido al estudio de la relación entre de los espacios verdes y la satisfacción social del usuario, la investigación cualitativa, de un carácter más personal y focalizado, podría ofrecer mejor información de cara al diseño, planificación y mantenimiento de las IV y las necesidades específicas de la comunidad local en las que se hayan insertos (Kaczynski \& Henderson, 2007).

Por último, en esta línea de usar instrumentos basados en las Tecnologías de la Información y la Comunicación, cabe resaltar cómo algunas investigaciones recientes destacan el papel que juega el uso de los SIG a la hora de plasmar cartográficamente las percepciones sociales sobre los servicios ecosistémicos que ofrecen la IV, a través de la realización de cartografía colaborativas que visualicen la dimensión territorial de dichas percepciones sociales respecto a los elementos paisajísticos (Martínez-Harms \& Balvanera, 2012), aspecto fundamental que debería tenerse muy en cuenta de cara al desarrollo de procesos de participación ciudadana basados en la idea de innovación y gobernanza social.

\section{La escala territorial}

A la hora de hacer referencia a las escalas de aplicación de los estudios que han servido de base para el diseño de esta investigación, resulta de espacial importancia remarcar el predominio de la escala local y municipal, especialmente intraurbana y barrial en la práctica totalidad $(90,6 \%)$ de los casos consultados. De manera generalizada un importante número de estudios se centra en una escala microterritorial, al centrar su análisis de manera concreta en parques de carácter barrial de mayor o menor tamaño. También destacan aquellos casos en los que se comparan el uso o las implicaciones sociales de diversos parques de una ciudad, atendiendo a sus características de tamaño, frecuencia de uso, facilidad en el acceso, etc...Sin embargo la escala metropolitana $(3,1 \%)$ resulta prácticamente ausente, siendo inexistentes los casos de estudio abordados desde la escala regional. 
De manera generalizada los estudios utilizados se han centrado en el análisis de las implicaciones sociales de los espacios verdes locales o barriales, dejando de lado el enfoque territorial metropolitano que, ante las características de las IV, es el más adecuado para entender el modo en que los espacios libres que las componen actúan para conseguir percepciones ciudadanas favorables respecto el bienestar socioambiental dentro de las áreas metropolitanas. En este sentido resulta evidente que por el carácter extenso en el territorio de las IV, se hace necesario la aplicación de unas escalas de análisis más amplias que permitiese analizar de forma global su impacto social d a nivel metropolitano.

\section{Antecedentes teóricos y epistemológicos en el análisis de la dimensión social de las IV}

Cómo se ha reseñado, la IV es una parte esencial de la planificación urbana y metropolitana y juega un papel clave en la conservación de la calidad ambiental del entorno urbano, permite a la población disfrutar de actividades recreativas y de ocio de diferente tipo, aliviar el nivel de estrés y resulta fundamental en el proceso de sociabilización (Mansor, Said \& Mohamad, 2012). Las actividades sociales que se desarrollan en la IV, ya sea de una manera activa o pasiva, proporciona un bienestar físico directo e induce mediante procesos psicológicos a un bienestar cognitivo y social (Maller et al., 2005).

Para la planificación territorial y urbanística, la IV resulta un instrumento óptimo para minimizar los impactos ecológicos y sociales del crecimiento urbano difuso, el consumo acelerado de suelo y la fragmentación del espacio libre. Sin embargo a pesar de esta importante vinculación entre las IV, los elementos que la componen y el bienestar social y físico de los usuarios, se debe tener en cuenta que la literatura científica que analiza la vinculación entre los factores ha tenido una corta y reciente trayectoria (Crilley, Weber \& Taplin, 2012). Partiendo de estas premisas, y de la indagación bibliográfica desarrollada, puede considerar que los beneficios de las IV desde un punto de vista social se pueden dividir en cinco categorías socioambientales principales.

\section{Desarrollo de la interacción y la cohesión social}

La interacción social y las actividades físicas dentro de los espacios verdes tienen un impacto positivo en la calidad de vida de los residentes. Estos espacios forman una parte fundamental del paisaje de cualquier ciudad y están estrechamente vinculados con la identidad colectiva de la comunidad (Inglis, Deery \& Whitelaw, 2008; Martí, et al., 2020; Zwierzchowska et al., 2019). Entendiendo que los espacios verdes son uno de los componentes de la IV, resulta evidente que dicha infraestructura es una parte crucial del tejido urbano desde el punto de vista social ya que determina en gran manera las percepciones de los residentes respecto a su bienestar físico, cognitivo y social.

Los resultados de las diferentes investigaciones analizadas sugieren que los residentes perciben la IV como espacios donde tienen contacto con la naturaleza y que satisface las necesidades de interacción social y comunitaria (Lo \& Jim, 2010). Así los espacios que conforman la IV permiten una gran diversidad de tipología de contactos e interacción social para los ciudadanos a partir del estímulo que supone la calidad ambiental que estos espacios ofrecen a los usuarios (Mansor, 
Said \& Mohamad, 2012). Del mismo modo se puede afirmar que tanto el origen como la frecuencia del uso del espacio verde son los aspectos que están más fuertemente relacionados con el apego de los residentes a los parques urbanos (Peters, Elands \& Buijs, 2010).

En general desde un punto de vista social, los patrones de uso de los espacios verdes se relacionan con las características demográficas y socioeconómicas de sus usuarios, la distribución y la accesibilidad, así como la calidad percibida y el valor del equipamiento de las áreas verdes (Chiesura, 2004). De este modo las percepciones sobre el uso de los espacios verdes estarían vinculadas a la edad, el género, los ingresos, la educación y la pertenencia a grupos étnicos o culturales (Ujang, Moulay, \& Zakariya, 2015).

Mientras que los jóvenes usan los espacios verdes con fines deportivos o de reunión, los adultos y los ancianos prefieren acceder a ellos para pasear, salir con niños o contemplar la naturaleza (Shan, 2014).

A su vez son numerosos los trabajos que inciden en el papel que la IV juega en el desarrollo de las relaciones entre la calidad de los parques, el carácter de las visitas y el alcance de los vínculos sociales en el vecindario (Dipeolu, Ibem \& Fadamiro, 2020; Huang, 2010). Se puede afirmar que la satisfacción del visitante depende en parte de los activos tangibles del parque, como la calidad del servicio (Tonge \& Moore, 2007), así como de la percepción y satisfacción subjetiva que desarrolla el visitante en su uso y disfrute del espacio verdes (Anderson et al., 2000).

Del mismo modo las características físicas de los espacios verdes determinan en gran medida las percepciones de bienestar y satisfacción de los usuarios (Mohamed \& Othman, 2012; Venkataramanan et al., 2019). Este aspecto resulta fundamental para generar percepciones positivas respecto al mayor bienestar social. Incluso algunos estudios afirman que potencialmente los espacios verdes incidirían en la reducción de los comportamientos sociales violentos, contribuyendo a un incremento en el sentimiento de aprecio y sentido de lugar, y por lo tanto jugarían un papel fundamental en el fomento de una mayor sentimiento de cohesión e identidad social en aquellos barrios que disponen de estos elementos urbanos (Dempsey, Brown \& Bramley, 2012).

\section{Salud y bienestar social}

De una manera más cuantitativa que en el caso anterior numerosos estudios internacionales han analizado la relación en el papel de las IV con respecto a la salud mental y el bienestar de las comunidades. Los espacios públicos abiertos como parques, espacios verdes e instalaciones de ocio pueden ayudar a promover un modelo de vida saludable en el espacio urbano al brindar oportunidades para la realización prácticas física, deportivas y actividades relacionadas con el ocio.

Esta capacidad de promover el bienestar físico y psicológico también se relaciona con una mayor facilidad a la hora de fomentar la integración social de los usuarios (Fermino et al., 2013). La cercanía a los espacios verdes amplían la posibilidad de desarrollar de actividades físicas, producen un mayor beneficio para la salud mental y física y reduciendo el riesgo de desarrollar enfermedades crónicas (Pirnat \& Hladnik, 2019; Warburton, Nicol \& Bredin, 2006). 
Son numeroso los estudios que han analizado las posibilidades que los espacios verdes brindan en la escala local para la realización de diferentes tipos de actividades de ocio y en el fomento de la actividad física (McCormack et al., 2010). Kaczynski \& Henderson (2007) analizaron que la proximidad a los parques estaba asociada con un aumento de la actividad física. Así el bienestar físico se encuentra relacionado con el comportamiento de los residentes hacia la posibilidad de realizar actividades recreativas en los espacios disponibles en la red de IV. Actividades como correr, caminar o jugar contribuyen a un mayor sentimiento de salud (Booth et al., 2000; Venkataramanan et al., 2019). A su vez el acceso a parques y entornos naturales cercanos se relaciona con una mejor salud mental, reducción del estrés y mejora de la salud física (Payne et al., 2005).

En otros casos, el mejor acceso a espacios verdes en las ciudades se correlaciona con una mayor esperanza de vida (Takano, Nakamura \& Watanabe, 2002) y mejor recuperación de enfermedades, así como con una mayor autopercepción de salud (Van den Berg et al., 2010).

Sin embargo, aunque la evidencia de la mayoría de los estudios sugiere que los espacios verdes tienen efectos beneficiosos para la salud, se debe tener en cuenta que establecer una relación estadísticamente causal resulta muy difícil (Lee \& Maheswaran, 2010). En este sentido el carácter excesivamente local y cualitativo de la práctica totalidad de los estudios desarrollados explicaría esta dificultad para hacer generalizables estos resultados.

\section{Integración y vulnerabilidad social}

Una de las funciones más importantes que se deriva de la función social de la IV es su relación con la mejora de la integración de grupos socialmente vulnerables (Maas et al., 2009), siendo fundamental el papel de estos espacios para mejorar la integración de la minorías étnicas, especialmente entre los grupos más jóvenes (Ravenscroft \& Markwell, 2000).

Se puede afirmar que la población inmigrante usa de forma grupal mucho más que la población nativa los parques urbanos (Kaergård, 2010). Las interacciones en la vida diaria entre personas de distinto origen étnico son un modo de crear cohesión social, y generar una base para los vínculos sociales entre los individuos (Marshall \& Stolle, 2004).

A su vez las interacciones con otras personas ayudan a los miembros de colectivos en riesgo de vulnerabilidad social a participar en la sociedad y crear sentimientos de aceptación (Putnam, 2000). En estos colectivo vulnerables el acceso a espacios verdes urbanos contribuye a aumento de la socialización de los residentes urbanos (Dai, 2011).

No obstante al igual que en el caso de la relación espacios verdes/salud, y debido a los obstáculos metodológicos y teóricos, algunos estudios advierten que no se deben sacar conclusiones tempranas sobre la situación del contacto en los espacios verdes porque la forma y la calidad del contacto en la vida diaria suele tener un carácter limitado y superficial (Dixon \& Durrheim, 2004). Por lo tanto, el uso muy común de un espacio no implica necesariamente una interacción considerable (Maoz, 2002).

Sin embargo, a pesar de esta afirmación sobre el carácter informal y superficial de las interacciones en los espacios verdes para estimular la cohesión social, otros estudios afirman que tales 
interacciones influyen positivamente en la cohesión social ya que ofrecen la oportunidad para el desarrollo de altos niveles de interacción social entre personas de diferentes orígenes sociales y étnicos (Rasidi, Jamirsah \& Said, 2012).

De este modo, una gran parte de los estudios afirman que los parques urbanos tienen más potencial para la inclusión social que en cualquier otro espacio público, especialmente porque el acceso y oportunidad de desarrollar actividades colectivas es mayor que en otros eso espacios urbanos. Además, se desarrolla una mayor sentimiento de unidad y cohesión social entre las personas que interactúan en actividades de ocio, especialmente si son deportivas (Konijnendijk et al., 2013; Ward, et al., 2019).

En esta línea, se incide en el papel que la presencia de la vegetación en los espacios verdes juega a la hora de reducir el temor a la delincuencia, la aparición de comportamientos de carácter antisocial (Kuo \& Sullivan, 2001), así como reducir el estrés y minimizar la incidencia de actividades delictivas (Donovan \& Prestemon, 2010). También se refleja una relación entre la calidad del mantenimiento de la vegetación en los parques, la existencia de bajos los índices de delincuencia a partir del fortalecimiento de los vínculos comunitarios que desarrollan los espacios verdes (Rij, Dekkers \& Koomen, 2008). Por lo tanto, se puede concluir que un valor social fundamental de los espacios verdes consiste en reunir a miembros de diferentes orígenes sociales y fomentar su interacción social aunque sea de manera fugaz y superficial (Barbosa et al., 2007).

Sin embargo, a pesar de estos resultados, pocos estudios utilizan indicadores para medir la cohesión social que permitan generar modelos explicativos concluyentes sobre cómo los espacios verdes pueden mejorar la cohesión social (i.e. Fan, Das \& Chen, 2011). De nuevo el carácter excesivamente microsocial de la mayoría de los estudios desarrollados dificulta hacer este tipo de análisis y reflexiones.

\section{Diseño, planificación e innovación social}

Los espacios verdes de la ciudad se deben planificar y gestionar desde una óptica en la que prime el beneficio público (Rall, Hansen \& Pauleit, 2019). Por ello, la gestión de los espacios verdes debe partir de un buen conocimiento de las expectativas y actitudes de los usuarios (Jennings et al., 2016; Voghera \& Giudice, 2019). Así una buena gestión de los espacios verdes de la ciudad requiere que los gerentes tengan un profundo conocimiento de las expectativas y actitudes de los usuarios con respecto a los servicios del parque. Los planificadores y los gestores públicos deben manejar información actualizadas sobre las preferencias sociales que se desarrollan sobre cada parque a la hora de planificar el diseño o administración de los mismos (Lamond \& Everett, 2019; Yung, Ho \& Chan, 2017). En este sentido se debe tener en cuenta que las preferencias y las actitudes de los usuarios pueden variar según el tipo y ubicación de los parques (Willems et al, 2019).

En esta línea hay estudios que resaltan la importancia del adecuado diseño y mantenimiento de los espacios verdes para el desarrollo de todo su potencial de cara a favorecer las interacciones y relaciones sociales (Kazmierczak, 2013). De ahí la importancia de diseñar y mantener estos espacios en cooperación con los usuarios locales utilizando la idea de innovación social de cara a aumentar la frecuencia de uso (Moulay, Ujang \& Said, 2017). 
Para estimular la interacción social en los espacios verdes, las estrategias basadas en la innovación social hacen partícipe a la comunidad en la adopción de decisiones que implique su gestión. Al permitir esta implicación en la gestión y mantenimiento por parte del vecindario se produce un sentimiento de identificación con el lugar y apropiación del espacio verde por parte de los individuos y un aumento en el compromiso de su mantenimiento y cuidado (Colding \& Barthel, 2013; Rall, Hansen \& Pauleit, 2019).

Se ha demostrado que los parques con un adecuado mantenimiento de su equipamiento mejoran el sentimiento de bienestar general de sus usuarios (Jackson, 2003). Atributos como el tamaño del parque (Giles-Corti et al., 2005), la presencia de campos deportivos bien cuidados (Floyd et al., 2008) o áreas boscosas, caminos y aceras en buen estado (Chan, Chau \& Leung, 2017), promueven una mayor uso del parque, mientras que la presencia de basura, vandalismo y baños sucios puede disuadir su uso (Gobster, 2002). De hecho los espacios inseguros o mal iluminados y mantenidos pueden producir una falta de interés en su uso incluso cuando están ubicados en el ámbito cotidiano del usuario (Powell, Martin \& Chowdhury, 2003).

\section{Distancia, accesibilidad y frecuencia de uso}

Otro importante foco de análisis en estos estudios es la relación existente entre distancia y accesibilidad, entendida cómo proximidad y tiempo de acceso a los espacios verdes (Pirnat \& Hladnik, 2019; Pitarch-Garrido, Magraner-Fajardo \& Gallego-Zornoza, 2017), con su frecuencia y tipología de uso. Diversos estudios parten del supuesto de que las distancias más cortas desde el lugar de residencia a los espacios verdes aumentan la accesibilidad y la probabilidad de que las personas usen dicho ámbito (Peschardt \& Stigsdotter, 2013).

Sin embargo, contrariamente otros estudios reflejan que la distancia a los espacios verdes no es un factor limitante para el uso de estos espacios (Schipperijna et al., 2010). De hecho la distribución y la accesibilidad puede variar debido a factores tales como el ingreso familiar, las características del mercado de la vivienda y la etnia (Heinen, Perkins \& Roy, 2009). Del mismo modo la distancia a los parques y los espacios abiertos se asocia de manera inversa con el uso y el comportamiento de la actividad física (Reyes-Paecke \& Figueroa Aldunce, 2010), lo que podía sugerir que la creación de más parques en los vecindarios a poca distancia de la mayoría de los residentes podría alentar un incremento en la realización de actividad física en la población. El acceso al transporte público también se identificó como un facilitador de la actividad física y el uso de los parques (McCormack et al. 2010).

Esta clara relación entre cercanía al espacio verde y su mayor uso y disfrute vienen reflejado tanto en estudios de carácter cuantitativo (Giles-Corti et al., 2005), como por estudios cualitativos (Kaczynski \& Henderson, 2007) y reafirma la importancia de que la IV esté compuesta por una variada red de componentes por todo el espacio metropolitano que fomente un mayor uso ciudadano.

Por último cabe reseñar aquellos estudios que plantean cómo los patrones de uso, accesibilidad y distancia de estos espacios favorecen, al menos potencialmente, una mayor interacción entre residentes de diferentes entornos socioeconómicos (Peters, Elands, \& Buijs, 2010). Todos estos aspectos hasta aquí referenciados, adquieren mayor importancia, ante la cada vez mayor 
expansión territorial y carácter difuso del hecho urbano y metropolitano (Krellenberg, Welz \& Reyes-Päcke, 2014).

\section{Discusión: Perspectivas e instrumentos para el análisis de la satisfacción socioambiental en el espacio metropolitano}

Tras analizar los resultados en las tres dimensiones planteadas y desagregar los principales ejes temáticos que abordan los estudios que relacionan la dimensión social de los espacios que conforman la IV, se puede considerar que las decisiones de carácter metodológico que se ha puesto en práctica determinan de manera directa inciden las cuestiones de índole epistemológico.

En el primer caso resulta evidente la importancia de implementar una escala territorial mayor a la local para poder entender de manera holística e integrada la relación entre la satisfacción medioambiental de las IV en el ámbito metropolitano. Es decir, atendiendo a la dimensión de red territorial integrada y multifuncional de los espacios en el área metropolitana que caracteriza a la IV, es necesario que el análisis socioambiental que se diseñe abarque la mayor realidad territorial posible del espacio analizado. En este caso los estudios deben plantearse desde un ámbito poliadministrativo que resulte representativo de la complejidad del territorio analizado. Así sería fundamental para captar globalmente la dimensión social de la IV realizar una selección de ámbitos territoriales que tenga un valor muestral de la diversidad socioambiental del ámbito metropolitano analizado.

En cuanto a la metodología, los estudios analizados reflejan al tratar varias de las temáticas abordadas que los medios cuantitativos presenta ciertas limitaciones para explicar la causalidad estadística en las relaciones entre la dimensión social y ambiental de estos espacios (i.e. Fan, Das \& Chen, 2011). Por otro lado la literatura académica claramente marca los medios cualitativos como los más adecuados para captar y describir las percepciones sociales subjetivas. Sin embargo los instrumentos cualitativos analizados, a pesar de mostrar la dimensión interna que explica la satisfacción socioambiental que producen las IV, muestran una dificultad a la hora de generalizar sus resultados debido al carácter esencialmente local que caracteriza su enfoque (Flyvbjerg, 2006).

Por tanto, enlazando con lo tratado inicialmente en este apartado, se hace necesario desarroIlar estrategias que nos permitan analizar las subjetividades y percepciones sociales sobre la IV a escala metropolitana. Para ello a la hora de seleccionar los ámbitos de estudio dentro del espacio metropolitano se debe superar el enfoque local. Para ello la realización de muestreos intencionados (Barbour, 2001), tanto en los grupos sociales diana cómo en los ámbitos territoriales para seleccionar donde aplicar los medios cualitativos así como el uso y representación de los resultados a través de SIG participativos (Brown \& Fagerholm, 2015) se plantean como recursos que pueden abrir muchas posibilidades.

Para este fin, puede ser de gran eficacia la difusión de encuestas a través de redes sociales y contactos directo con colectivos sociales que representen un amplio espectro de puntos de vista 
vinculados por su uso social o profesional a las tipologías de espacios verdes seleccionados, sin tener como objetivo una muestra estadísticamente representativa es una opción metodológica que se debe tener en cuenta (De Vreesea et al, 2016). De hecho el uso de instrumentos de Google Forms y su difusión mediante correo electrónico y grupos de WhatsApp ha sido ya de otras investigaciones centradas en captar las percepciones y grado de satisfacción de los usuarios (e.i. Villímar Rodríguez et al., 2016).

Así se puede afirmar que los últimos años se está produciendo un fuerte desarrollo de las encuestas realizadas a través de internet. En este contexto, trabajar con instrumentos online resulta muy atractivo, tanto por el bajo coste asociado a la fase de recogida de datos, como por su rapidez a la hora de tramitar la información (De Marchis, 2012; De Vreesea et al, 2016). Sin embargo, no se puede obviar que las encuestas online presentan inconvenientes relacionados con el desigual uso de las nuevas tecnologías en los distintos segmentos de la población (Díaz de Rada, 2012). Por otro lado cómo ya se ha comentado, existe una creciente dificultad para conseguir un marco muestral apropiado que permita la aplicación de técnicas de muestreo aleatorio probabilístico. A pesar de ello, en un entorno social cada vez más virtualizado, dinámico y caracterizado por la inmediatez (Rosa, 2011) se está recurriendo cada vez más a estas técnicas de recogidas de información con el objetivo de dar respuesta a dos de los principales problemas que amenazan en los últimos años la investigación mediante encuestas: (i) la dificultad creciente para contactar y (ii) asegurar la participación de la población objeto de estudio.

Tras esta reflexión sobre las estrategias metodológicas más adecuadas para analizar la dimensión social de la IV en la escala metropolitana se puede concluir que aspectos cómo las percepciones sociales de la ciudadanía sobre los espacios que la componen resultan fundamentales para asegurar bienestar socioambiental de la ciudadanía en unos ámbitos urbanos cada vez más extensos, difusos y complejos. Así de cara a la labor de planificación y diseño de la IV, el adecuado pulso sobre las demandas ciudadanas sobre los espacios que soportan la función socioambiental resulta un aspecto clave (Jennings et al., 2016). Aspectos cómo las características del diseño de estos espacios, su mantenimiento, infraestructuras instaladas, iluminación, cercanía y acceso se han mostrado cómo fundamentales a la hora de entender las percepciones que se desarrollan sobre estos ámbitos. Si a ello se añade su papel cómo ámbitos para fomentar la convivencia social y mejora del bienestar físico y mental, resulta evidente la conveniencia de generar instrumentos que permitan dar información a la administración pública a la hora de tomar decisiones sobre el diseño y mantenimiento de la red de espacios libres que componen la IV. De ahí la importancia de analizar las subjetividades sociales que el ciudadano generar hacia los ámbitos territoriales de la IV (Kothencz \& Blaschke, 2017; van Vliet \& Hammond, 2020).

En este sentido la línea a seguir debe pasar por un mayor desarrollo de la gobernanza ciudadana que signifique in incremento de la implicación en la gestión y mantenimiento comunitario y vecinal de cara a aumentar el sentimiento de identificación con el espacio verde y un aumento en el compromiso de su mantenimiento y cuidado (Colding \& Barthel, 2013; Lamond \& Everett, 2019; Rall, Hansen \& Pauleit, 2019). Con ello, partiendo de la idea de innovación socioecológica (Dennis, Armitage, \& James, 2016) se plantea la importancia conseguir una gestión de los espacios verdes más descentralizada, adaptada a las características concretas e individualizada de cada espacio urbano y metropolitano que permita una gestión más adaptativa y resiliente según la diversidad de realidades locales del espacio metropolitano. 


\section{Referencias bibliográficas}

AGUILERA BENAVENTE, F., RODRÍGUEZ ESPINOSA, V. \& GÓMEZ DELGADO, M. Definición de infraestructuras verdes: una propuesta metodológica integrada mediante análisis espacial. Documents D’AnàLisi GeogràFica, 2018, Vol 64, N², p. 313-337.

ANDERSON, D., NICKERSON, R., STEIN, T., \& LEE, M. Planning to provide community and visitor benefits from public lands. In W. Gartner, \& D. Lime (editores). Trends in outdoor recreation, leisure and tourism. Wallingford, New Zealand: Ediciones CABI Publishing, 2000, p. 197-211.

ANGUELOVSKI, I., IRAZÁBAL ZURITA, C. \& CONNOLLY, J.J. Grabbed Urban Landscapes: Socio-spatial Tensions in Green Infrastructure Planning in Medellín. 2019, Int. J. Urban Reg. Res., 43, p. 133156. doi:10.1111/1468-2427.12725

BARBOSA, O., TRATALOS, J.A., ARMSWORTH, P.R., DAVIES, R.G., FULLER, R.A., JOHNSON, P., \& GASTON, K.J. Who benefits from access to green space? A case study from Sheffield, UK. Land scape and Urban planning, 2007, Vol 83, No 2-3, p.187-195.

BARBOUR R. S. Checklists for improving rigour in qualitative research: a case of the tail wagging the dog? BMJ, 2001, Vol 322, No 7294, p.1115-1117.

BARNHILL, K. \& SMARDON, R. Research Article: Gaining Ground: Green Infrastructure Attitudes and Perceptions from Stakeholders in Syracuse, New York. Environmental Practice, 2012, Vol 14 No 1, p.6-16.

BAXENDALE, C. A. \& BUZAI, G. D. Modelos urbanos e infraestructura verde en ciudades de América Latina. Análisis en la ciudad de Buenos Aires. Revista Huellas, 2019, Vol 23, № 2, p.79-106..

BROWN, G., SCHEBELLA, M. FAITH, \& WEBER, D. Using participatory GIS to measure physical activity and urban park benefits. Landscape and urban planning, 2014, Vol 121, p. 34-44.

BROWN, G. \& FAGERHOLM, N. Empirical PPGIS/PGIS mapping of ecosystem ser-vices: a review and evaluation. Ecosystem Services, 2015, Vol 13, p.119-133,

BOOTH, M., OWEN, N., BAUMAN, A., CLAVISI, O., \& LESLIE, E. Social cognitive and perceived environment influences associated with physical activity in older Australians. Preventive Medicine, 2000, Vol 3, p.15-22.

CANTÓ, M. La ordenación de la Infraestructura Verde en el sudeste Ibérico (Comunidad Valenciana, España). Cuadernos de Biodiversidad, 2014, No 45, p.10-22.

CHAN, S., CHAU, C. \& LEUNG, T. On the study of thermal comfort and perceptions of environmental features in urban parks: A structural equation modeling approach. Building and Environment, 2017, Vol 122, p.171-183. 
CHIESURA, A. The role of urban parks for the sustainable city. Landscape and Urban Planning, 2004, Vol 68, N01, p.129-138.

COLDING, J., \& BARTHEL, S. The potential of 'urban green commons' in the resilience building of cities. Ecological Economics, 2013, Vol 86, p.156-166.

CRILLEY, G., WEBER, D., \& TAPLIN, R. Predicting visitor satisfaction in parks: comparing the value of personal benefit attainment and service levels in Kakadu National Park, Australia. Visitor Studies, 2012, Vol 15, No2, p.217-237.

DAI, D. Racial/ethnic and socioeconomic disparities in urban green space accessibility: where to intervene? Landscape and Urban Planning, 2011, Vol 102, p.234-244.

DE MARCHIS, G. P. La validez externa de las encuestas en la web. Amenazas y su control. Estudios sobre el mensaje periodístico, 2012, Vol. 18, p. 263272. Madrid, Servicio de Publicaciones de la Universidad Complutense.

DEMPSEY, N., BROWN, C., \& BRAMLEY, G. The key to sustainable urban development in UK cities? The influence of density on social sustainability. Progress in Planning, 2012, Vol 77, No3, p.89-141.

DENNIS, M., ARMITAGE, R.P. \& JAMES, P. Social-ecological innovation: adaptive responses to urban environmental conditions. Urban Ecosystems, 2016, Vol 19, N³, p.1063-1082.

DE VREESEA, R., LEYS, M., FONTAINE, C. \& DENDOCKER, N. Social mapping of perceived ecosystem services supply - The role of social landscape metrics and social hotspots for integrated ecosystem services assessment, landscape planning and management. Ecological Indicators, 2016, Vol 66, p.517-533.

DÍAZ DE RADA, V. Ventajas e inconvenientes de la encuesta por internet. Papers. Revista de Sociología, 2012, Vol. 97 (1), p. 193-223. http://dx.doi.org/10.5565/rev/papers/v97n1.71

DIPEOLU , A.A., IBEM, E.O. \& FADAMIRO, J. A. Influence of green infrastructure on sense of community in residents of Lagos Metropolis, Nigeria. Journal of Human Behavior in the Social Environment, 2020, Vol 30 (6), p. 743-759, DOI: 10.1080/10911359.2020.1740853

DIXON, J., \& DURRHEIM, K. Dislocating identity: desegregation and the transformation of place. Journal of Environmental Psychology,2004, Vol 24, p.455-473.

DONOVAN, G., \& PRESTEMON, J. The Effect of Trees on Crime in Portland, Oregon. Environment and Behavior, 2010, Vol 44, No1, p.3-30.

FAN, Y., DAS, K., \& CHEN, Q. Neighborhood green, social support, physical activity, and stress: Assessing the cumulative impact. Health \& Place 17, 2011, p.1202-1211. 
FERIA-TORIBIO, J. \& SANTIAGO RAMOS, J. Naturaleza y ciudad. Perspectivas para la ordenación de la infraestructura verde en los planes territoriales metropolitanos en España. BAGE, 2017, N074, p.117-141.

FERMINO, R., SIQUEIRA, R., CURI, P. \& CAZUZA DE FARIAS, J. Perceived environment and public open space use: a study with adults from Curitiba, Brazil. International Journal of Behavioral Nutrition and Physical Activity, 2013, Vol 10 No 35, p.1-10.

FLOYD, M., SPENGLER, J., MADDOCK, J., GOBSTER, P. \& SUAU, L. Park-based physical activity in diverse communities of two U.S. cities. An observational study. American Journal of Preventive Medicine, 2008, Vol 34, p.299-305.

FLYVBJERG, B. "Five Misunderstandings About Case-Study Research". Qualitative Inquiry, 2006, Vol 12, No2, p.219-245.

GILES-CORTI, B., TIMPERIO, A., BULL, F. \& PIKORA, T. Understanding physical activity environmental correlates: increased specificity for ecological models. Exercise and Sport Science Reviews, 2005, Vol 33, p.175-181.

GOBSTER, P. Managing urban parks for a racially and ethnically diverse clientele. Leisure Sciences, 2002, Vol 24, p.143-159.

GREENHALGH, T. y PEACOCK, R. Effectiveness and efficiency of search methods in systematic reviews of complex evidence: audit of primary sources. British Medical Journal, 2005, Vol 331, p.1064-1065.

HEINEN, N., PERKINS, H., \& ROY, P. The political ecology of uneven urban green space: the impact of political economy on race and ethnicity in producing environmental inequality in Milwaukee. Urban Affairs Review, 2009, Vol 42, p.3-24.

HUANG, S. The Impact of Public Participation on the Effectiveness of, and Users Attachment to, Urban Neighbourhood Parks. Landscape Research, 2010, Vol 35, No5, p.551-562.

INGLIS, J., DEERY, M., \& WHITELAW, P. The Development of Place Attachment in Parks Sustainable Tourism, Queensland, Australian: Editorial Sustainable Tourism Cooperative Research Centre, 2008.

JACKSON, L. The relationship of urban design to human health andcondition. Landscape and Urban Planning, 2003, Vol 64, p.191-200.

JENNINGS, T., JEAN-PHILIPPE, S., WILLCOX, A., ZOBEL, J., POUDYAL, N. \& SIMPSON, T. The influence of attitudes and perception of tree benefits on park management priorities. Landscape and Urban Planning, 2016, Vol 153, p.122-128. 
KACZYNSKI, A. \& HENDERSON, K. Environmental Correlates of Physical Activity: A Review of Evidence about Parks and Recreation, Leisure Sciences, 2007, Vol 29, No4, p.315-354.

KAERGÅRD, N. Social cohesion and the transformation from ethnic to multicultural society: The case of Denmark. Ethnicities, 2010, Vol 10, No4, p.470-487.

KAZMIERCZAK, A. The contribution of local parks to neighbourhood social ties. Landscape and Urban Planning, 2013, Vol 109, No1, p.31-44.

KIM, D. \& SONG, S. The Multifunctional Benefits of Green Infrastructure in Community Development: An Analytical Review Based on 447 Cases. Sustainability, 2019, Vol 11, No 14, p.1-17.

KITCHENHAM, B. \& CHARTERS, S. Guidelines for Performing Systematic Literature Reviews in Software Engineering, Technical Report EBSE 2007-001. Journal of software engineering and aplications, 2007, Vol 8, No8.

KONIJNENDIJK, C., ANNERSTEDT, M., NIELSEN, A., \& MARUTHAVEERAN, S. Benefits of Urban Parks A systematic review. IFPRA, 2013, $\mathrm{N}^{\circ} 6$, p.10-12

KOTHENCZ, G. \& BLASCHKE, T. Urban parks: Visitors' perceptions versus spatial indicators. Land Use Policy, 2017, Vol 64, p.233-244.

KRELLENBERG, K., WELZ,J. \& REYES-PACKE, S. Urban green areas and their potential for social interaction. A case study of a socio-economically mixed neighbourhood in Santiago de Chile. Habitat International, 2014, Vol 44, p.11-21.

KUO, F., \& SULLIVAN, W. Environment and Crime in the Inner City: Does Vegetation Reduce Crime? Environment and Behavior, 2001, Vol 33, N013, p.343-367.

LAMOND, J \& EVERETT, G. Sustainable Blue-Green Infrastructure: A social practice approach to understanding community preferences and stewardship. Landscape and Urban Planning, 2019, Vol 191, 103639, https://doi.org/10.1016/j.landurbplan.2019.103639.

LEE, R., \& MAHESWARAN, A. The health benefits of urban green spaces: A review of the evidence. Journal of Public Health, 2010, Vol 3, No2, p.212-222.

LIBRETT, J., HENDERSON, K., GODBEY, G., \& MORROW, J. An introduction to parks, recreation, and public health: collaborative frameworks for promoting physical activity. Journal of physical activity \& health, 2007, vol 4, No1, p.1-13.

LO, A. \& JIM, C. Differential community effects on perception and use of urban greenspaces. Cities, 2010, Vol 27, Nº6, p.430-442.

LOVELL, S. \& TAYLOR, J. Supplying Urban Ecosystem Services through Multifunctional Green Infrastructure in the United States. Landscape Ecology, 2013, Vol 28, No 8, p.1447-1463. 
MAAS, J., VAN DILLEN, S., VERHEIJ, R. \& GROENEWEGEN, P. Social contacts as a possible mechanism behind the relation between green space and health. Health \& Place, 2009, Vol 15, $N^{\circ} 2$, p.586-595.

MANSOR, M., SAID, I. \& MOHAMAD, I. Experiential Contacts with Green Infrastructure's Diversity and Well-being of Urban Community. Procedia - Social and Behavioral Sciences, 2012, Vol 49, p.257-267.

MALLER, C., TOWNSEND, M., PRYOR, A., BROWN, P., \& LEGER, L. Healthy nature healthy people: 'Contact with nature' as an upstream health promotion intervention for populations. Health Promotion International, 2005, Vol 21, N01, p.45-54.

MAOZ, I. Is there contact at all? Intergroup interaction in planned contact interventions between Jews and Arabs in Israel. International Journal of Intercultural Relations, 2002, Vol 26, p.185-197.

MARTÍNEZ-HARMS, M. \& BALVANERA, P. Methods for mapping ecosystem services upply: a review. International Journal of Biodiversity Science, Ecosystem Services \& Management, 2012, Vol 8, p.17-25,

MARSHALL, M. \& STOLLE, D. Race and the City. Neighborhood Context and the Development of Generalized Trust. Political Behavior, 2004, Vol 26, p.125-154.

MARTÍ, P., GARCÍA-MAYOR, C., NOLASCO-CIRUGEDA, A. \& SERRANO-ESTRADA, L. Green infrastructure planning: Unveiling meaningful spaces through Foursquare users' preferences. Land Use Policy, 2020, Vol 97, 104641, https://doi.org/10.1016/j.landusepol.2020.104641.

MC CORMACK, G., ROCK, M., TOOHEY, A. \& HIGNELL, D. Characteristics of urban park sassociated with park use and physical activity: A review of qualitative research. Health \& Place, 2010, Vol 16, No4, p.712-726.

MOHAMED, N., \& OTHMAN, N. Push and pull factor: determining the visitor ssatisfactions at urban recreation area. Procedia-Social and Behavioral Sciences, 2012, Vol 49, p.175-182.

MOULAY, A., UJANG, N. \& SAID, I. Legibility of neighborhood parks as a predicator for enhanced social interaction towards social sustainability. Cities, 2017, Vol 61, p.58-64.

NEWELL, J., SEYMOUR, M., YEE, T., RENTERIA, J., LONGCORE, T., WOLCH, J., \& SHISHKOVSKY, A. Green Alley Programs: Planning for a Sustainable Urban Infrastructure? Cities, 2013, Vol 31, p.144-155.

PAYNE, L., ORSEGA-SMITH, E., ROY, M. \& GODBEY, G. Local park use and personal health among older adults: an exploratory study. Journal of Park and Recreation Administration, 2005, Vol 23, p.1-20.

PESCHARDT, K., \& STIGSDOTTER, U. Associations between park characteristics and perceived restorativeness of small public urban green spaces. Landscape and Urban Planning, 2013, Vol 112, p.26-39. 
PETERS, K., ELANDS, B. \& BUIJS, A. Social interactions in urban parks: Stimulating social cohesion? Urban Forestry \& UrbanGreening, 2010, Vol 9, No2 p.93-100.

PIRNAT, J. \& HLADNIK, D. A tale of two cities-From separation to common green connectivity for maintaining of biodiversity and well-being. Land Use Policy, 209, Vol 84, p. 252-259. https://doi. org/10.1016/j.landusepol.2019.03.011.

PITARCH-GARRIDO, M.D, MAGRANER-FAJARDO, F. \& GALLEGO-ZORNOZA. C. La naturaleza en la ciudad: la accesibilidad a los espacios verdes urbanos como medida de la calidad de vida. En Naturaleza, territorio y ciudad en un mundo global. Asociación de Geógrafos Españoles, 2017. p. 539-548.

POWELL, K., MARTIN, L. \& CHOWDHURY, P. Places to walk: convenience and regular physical activity. American Journal of Public Health, 2003, Vol 93, p.1519-1521.

PUTNAM, R. Bowling Alone: The Collapse and Revival of American Community. New York: Editorial: Simon and Schuster, 2000.

RASIDI, M., JAMIRSAH, N. \& SAID, I. Urban Green Space Design Affects Urban Residents' Social Interaction. Procedia - Social and Behavioral Sciences, 2012, Vol 68, p.464-480.

RAKHSHANDEHROO, M., MOHDYUSOF, M., TAHIRHOLDER, O. \& YUNOS, M. The Social benefits Of Urban Open Green Spaces: A Literature Review. Management Research and Practice, Research Centre in Public Administration and Public Services, Bucharest, Romania, 2015, Vol 7, Nº4, p.60-71.

RALL, E., HANSEN, R. \& PAULEIT, S. The added value of public participation GIS (PPGIS) for urban green infrastructure planning, Urban Forestry \& Urban Greening, 2019, Vol 40, p. 264-274, https:// doi.org/10.1016/j.ufug.2018.06.016.

RAVENSCROFT, N. \& MARKWELL, S. Ethnicity and the integration and exclusion of young people through urban park and recreation provision. Managing Leisure, 2000, Vol 5, No 3, p.135-150.

RICHARDS, D., \& THOMPSON, B. Urban ecosystems: A new frontier for payments for ecosystem services. People and Nature, 2019, Vol 1, No 2, p.249- 261.

REYES-PAECKE, S., \& FIGUEROA ALDUNCE, I. Distribución, superficie y accesibilidad de las áreas verdes urbanas en Santiago de Chile. Revista EURE, 2010, Vol 36, №2, p.89-110.

ROSA, H. Aceleración social: consecuencias éticas y políticas de una sociedad de alta velocidad desincronizada. Persona y Sociedad, 2011, Vol 25, N01, p.9-49.

SALVADOR-OLIVÁN, J., MARCO-CUENCA, G. \& ARQUERO-AVILÉS, R. Las revisiones sistemáticas en Biblioteconomía y Documentación: análisis y evaluación del proceso de búsqueda. Revista Española de Documentación Científica, 2018, Vol 41, №2, p.1-19. 
SCHIPPERIJNA, J., EKHOLMB, O., STIGSDOTTERA, U., TOFTAGERB, M., BENTSENA, P., \& KAMPERJØRGENSENB, F., Et al. Factors influencing the use of green space: results from a Danish national representative survey. Landscape and Urban Planning, 2010, Vol 95, No3, p.130-137.

SELMAN, P. Planning for landscape multifunctionality. Sustainability: Science, Practice and Policy, 2009, Vol 5, N², p.45-52,

SHAN, X. Socio-demographic variation in motives for visiting urban green spaces in a large Chinese city. Habitat International, 2014, Vol 41, p.114-120.

SULLIVAN, W., KUO, F. E., \& DEPOOTER, S. The fruit of urban nature: vital neighbourhood spaces. Environment and Behavior, 2004, Vol 36, No5, p.678-700.

SWAPAN, M., IFTEKHAR, M., \& LI, X. Contextual variations in perceived social values of ecosystem services of urban parks: A comparative study of China and Australia. Cities, 2017, Vol 61, p.17-26.

TAKANO, T., NAKAMURA, K., \& WATANABE, W. Urban residential environments and senior citizens' longevity in megacity areas: the importance of walkable green spaces. Journal of Epidemiology and Community Health, 2002, Vol 56, N012, p. 913-918.

TONGE, J., \& MOORE, S. Importance-satisfaction analysis for marine-parkhinter lands: a Western Australian case study. Tourism Management, 2007, N²22679, p.768-776.

UJANG, N., MOULAY, A. \& ZAKARIYA, K. Sense of Well-Being Indicators: Attachment to public parks in Putrajaya, Malaysia. Procedia - Social and Behavioral Sciences, 2015, Vol 202, p.487-494.

VAN DEN BERG, A., VAN WINSUM-WESTRA, M., DE VRIES, S., \& VAN DILLEN, S. Allotment gardening and health: A comparative survey among allotment gardeners and their neighbors without an allotment. Environmental Health, 2010, Vol 9, № 74, p.1-12.

VAN RIJ, E., DEKKERS, J., \& KOOMEN, E. Analysing the success of open space preservation in the Netherlands: the Midden-Delfland case. Tijdschrift voor economische en sociale geografie, 2008, Vol 99, No1, p.115-124.https://doi.org/10.1111/j.1467-9663.2008.00443.x

VAN VLIET, K. \& HAMMOND, C. Residents' perceptions of green infrastructure in the contemporary residential context: a study of Kingswood, Kingston-upon-Hull, England, Journal of Environmental Planning and Management, 2020, DOI: 10.1080/09640568.2020.1756757

VENKATARAMANAN, V. et al. A systematic review of the human health and social well-being outcomes of green infrastructure for stormwater and flood management. Journal of Environmental Management, 2019, Vol 246, p. 868-880, https://doi.org/10.1016/j.jenvman.2019.05.028.

VILlímar Rodríguez, A., FERmoso, A., PITA, C., \& CARDiel, G. Percepción de los médicos de atención primaria sobre la receta electrónica en el Servicio Madrileño de Salud. Revista de Calidad Asistencial, 2016, Vol 31 (6), p. 338-346. http://dx.doi.org/10.1016/j.cali.2016.01.008 
VOGHERA, A. \& GIUDICE, B. Evaluating and Planning Green Infrastructure: A Strategic Perspective for Sustainability and Resilience. Sustainability, 2019, Vol 11, 2726

WARBURTON, D., NICOL, C. \& BREDIN, S. Health benefits of physical activity: the evidence. Canadian Medical Association Journal, 2006, Vol 174, N6, p.801-809.

WARD, S. et al. Embedding social inclusiveness and appropriateness in engineering assessment of green infrastructure to enhance urban resilience. Urban Water Journal, 2019, Vol 16 (1), p. 56-67, DOI: 10.1080/1573062X.2019.1633674

WILLEMS, J., MOLENVELD, A., VOORBERG, W., \& BRINKMAN, G. Diverging Ambitions and Instruments for Citizen Participation across Different Stages in Green Infrastructure Projects. Urban Planning, 2020, 5(1), p. 22-32. doi:http://dx.doi.org/10.17645/up.v5i1.2613

YUNG, E., HO, W. \& CHAN, E. Elderly satisfaction with planning and design of public parks in high density old districts: An ordered logit model. Landscape and Urban Planning, 2017, Vol 165, p.3953.

ZWIERZCHOWSKA, I., FAGIEWICZ,K., PONIŻY, L., LUPA, P.\& MIZGAJSKI, A. Introducing nature-based solutions into urban policy - facts and gaps. Case study of Poznań. Land Use Policy, 2019, Vol 85, p. 161-175. https://doi.org/10.1016/j.landusepol.2019.03.025. 
